\title{
SURFACE REPRESENTATION OF POLYCRYSTAL PHYSICAL PROPERTIES: ALL CRYSTAL CLASSES, SIMPLE AVERAGE APPROXIMATION
}

\author{
O. RAYMOND*, L. FUENTES**, J. I. GÓMEZ** \\ *Instituto Superior Pedagógico Enrique José Varona, Marianao, Habana, Cuba \\ **Instituto de Cibernética, Matemática y Física, D \#353, Habana 10400, Cuba
}

(Received 5 September 1995; in final form 5 March 1996)

\begin{abstract}
Algorithms for polycrystal physical properties estimation are presented. Bunge's spherical harmonics treatment of surface representations, under simple average approximation, is applied. Specific formulae for so-called longitudinal magnitudes are given. Physical properties associated to tensors of second-, third- and fourth-rank are considered. All crystal and sample symmetries are covered.
\end{abstract}

KEY WORDS: Tensor properties, spherical harmonics, voigt average, crystal symmetry, sample symmetry.

\section{INTRODUCTION}

The calculation of physical properties in polycrystalline materials, starting from the corresponding properties of their constituent crystallites, is one of the important objectives in texture investigations. A general theoretical background for this problem has been given by Bunge (1982), who also wrote a pioneering work (1969) on ODF prediction of polycrystal mechanical properties. Recent developments in this field are associated to the use of orientation correlation and misorientation distribution functions, to self-consistent methods and presently to Matthies' (1994) geometrical mean approximation. The papers by Bunge (1989), Humbert (1991) and Mainprice (1994) may be representative of important moments in this working area.

A large number of specific studies have been performed in metallic (highly symmetric) systems. Special interest has been conferred to mechanical and magnetic properties. For the above mentioned cases, suitable algorithms and computer codes, ranging from simple averages to more sophisticated methods, have been established. However, in the case of low-symmetry materials, even for simple average estimations, we have not found a systematic presentation of such procedures.

Our purpose, with this work, is to contribute somewhat to a systematic treatment of polycrystal tensor properties. Specific algorithms for surface representation of properties associated to tensors of rank $r(2 \leq r \leq 4)$ are presented. Simple average approximation and Bunge's spherical harmonics ODF expansions are used. All crystal and sample symmetries are considered. 
THEORETICAL SUMMARY (for further details see Bunge, 1982)

Any macroscopic single-crystal tensor property $\mathrm{E}=\mathrm{Y} / \mathrm{X}$ may be represented by one or more scalar functions $\mathrm{E}(\mathrm{h})$ of the crystallographic direction $\mathrm{h}$. This gives place to the so-called surface representation (Nye, 1957). $\mathrm{X}$ and $\mathrm{Y}$ are measured quantities. The mentioned functions may be described by expansions in series of surface spherical harmonics of the crystal symmetry $\dot{\mathrm{K}}_{l}^{\mu}(\mathrm{h})$

$$
\mathrm{E}(\mathrm{h})=\sum_{l=0}^{r} \sum_{\mu=1}^{M(l)} e_{l}^{\mu^{i}} \mathrm{~K}_{l}^{\mu}(\mathbf{h})
$$

The coefficients $e_{l}^{\mu}$ characterize the property, $\mathrm{r}$ is the tensor's rank and $M(l)$ the number of linearly independent functions $\dot{\mathrm{K}}_{l}^{\mu}(\mathrm{h})$.

The coefficients $e_{l}^{\mu}$ may be calculated by means of the integral

$$
e_{l}^{\mu}=\oint \mathrm{E}(\mathrm{h}) \dot{\mathrm{K}}_{l}^{\mu}(\mathrm{h}) \mathrm{dh}
$$

Let us consider a macroscopically homogeneous polycrystalline sample with a texture described by the orientation distribution function (ODF)

$$
f(g)=\sum_{l=0}^{\infty} \sum_{\mu=1}^{M(l)} \sum_{v=1}^{N(l)} C_{l}^{\mu^{\nu}} \dot{\vec{T}}_{l}^{\mu v}(\mathrm{~g})
$$

The ODF, or likewise the coefficients $C_{l}{ }^{\mu \nu}$, characterize the volume fraction of crystallites in the neighborhood dg of the orientation $\mathrm{g} . \dot{\vec{T}}_{l}^{\mu v}(\mathrm{~g})$ are symmetry-adapted three-dimensional spherical harmonics.

If the crystallites of the considered sample show the property $E(h)$, then the polycrystal simple average property $\bar{E}$ will be a function of the sample direction $y$ and may be calculated, in the so-called Voigt's approximation, by the general formula

$$
\overline{\mathrm{E}}(\mathrm{y})=\sum_{l=0}^{r} \sum_{v=1}^{N(l)} \bar{e}_{l}{ }^{v} \dot{\mathrm{K}}_{l}^{v}(\mathrm{y})
$$

$N(l)$ is the number of linearly independent functions $\dot{\mathrm{K}}_{l}^{\nu}(\mathrm{y})$ of the sample symmetry. The acceptance of the Voigt approximation is equivalent to the assumption of a constant value for the variable $X$ over the entire polycrystal.

The coefficients $\bar{e}_{l}^{\nu}$ summarise the effects of both single crystal property and crystallite orientation distribution, characterized by $e_{l}{ }^{\mu}$ and $C_{l}{ }^{\mu v}$ respectively. They are calculated by

$$
\bar{e}_{l}^{\nu}=\frac{1}{2 l+1} \sum_{\mu=1}^{M(l)} C_{l}^{\mu \nu} e_{l}^{\mu}
$$

The symmetric functions in (1), (3) and (4) used in this work are those real functions described by Raymond et. al..

If the orientation distribution is rotationally symmetric about the Z-axis of the sample coordinate system, then the expansion (3) coincides with that of the fibre axis inverse pole figure $R(h)$. 


$$
f(\mathrm{~h})=\mathrm{R}(\mathrm{h})=\sum_{l=0}^{\infty} \sum_{\mu=1}^{M(l)} C_{l}^{\mu} \dot{K}_{l}^{\mu}(\mathrm{h})
$$

$C_{l}{ }^{\mu}$ characterizes the frequency distribution of crystal directions relative to the sample fibre axis. In this case, the property of the polycrystalline material is likewise rotationally symmetric about the Z-axis and

$$
\overline{\mathrm{E}}(\mathrm{y})=\overline{\mathrm{E}}(\phi)=\sum_{l=0}^{r} \bar{e}_{l} \bar{P}_{l}(\phi)
$$

with

$$
\bar{e}_{l}=\frac{1}{4 \pi} \sqrt{\frac{2}{2 l+1}} \sum_{\mu=1}^{M(l)} C_{l}^{\mu} e_{l}^{\mu}
$$

\section{TENSOR PROPERTIES OF SECOND RANK}

Consider a physical property described by a symmetric tensor of second rank $\mathrm{E}$. Examples of such properties are electric and magnetic susceptibilities, electric conductivity and thermal expansion. The so-called longitudinal magnitude of the analyzed property, in a given direction may always be described by a surface $\mathrm{E}(\mathrm{h})$. The analytical expression of $\mathrm{E}(\mathrm{h})$, relative to its principal axes, is

$$
E(h)=E_{11} h_{1}^{2}+E_{22} h_{2}^{2}+E_{33} h_{3}^{2}
$$

where $h_{i}$ are the direction cosines of the crystal direction $h$.

With respect to such properties all crystals have at least orthorhombic symmetry. Equation (9) may be expressed, according to (1), by means of orthorhombic spherical surface harmonics. The coefficients $e_{l}^{\mu}$ and the functions $\dot{K}_{l}^{\mu}(\mathrm{h})$ are summarized in Table 1.

Table 2 shows the coefficients $\bar{e}_{l}^{\nu}$ and the functions $\dot{K}_{l}^{\nu}(\mathrm{y})$ corresponding to equations (4) and (5) for a polycrystal, with all common sample symmetries taken into account. Subindex $t$ identifies triclinic coefficients and functions

For fibre textures, according to (7) and (8) one obtains

$$
\begin{aligned}
& \bar{e}_{0}=\frac{\sqrt{2}}{4 \pi} C_{0}^{1} e_{0}^{1}=\frac{1}{\sqrt{2 \pi}} e_{0}^{1} \\
& \bar{e}_{2}=\frac{1}{4 \pi} \sqrt{\frac{2}{5}}\left[C_{2}^{1} e_{2}^{1}+C_{2}^{2} e_{2}^{2}\right]
\end{aligned}
$$

\section{TENSOR PROPERTIES OF THIRD RANK}

Properties represented by symmetric tensors of third rank (specifically piezoelectricity) are exclusive of systems without centre of inversion. Here the longitudinal surface is described by 
Table 1 Second-rank tensors. Single crystals.

\begin{tabular}{l|c|c|c}
\hline$l$ & $\mu$ & $e_{l}{ }^{\mu}$ & $\dot{K}_{l}^{\mu}(h)^{*}$ \\
\hline 0 & 1 & $e_{0}^{1}=\frac{2}{3} \sqrt{\pi}\left(\mathrm{E}_{11}+\mathrm{E}_{22}+\mathrm{E}_{33}\right)$ & $\overline{\mathbf{K}}_{0}^{0}$ \\
\hline 2 & 1 & $e_{2}^{1}=\frac{2}{3} \sqrt{\frac{\pi}{5}}\left(2 \mathrm{E}_{33}-\mathrm{E}_{11}-\mathrm{E}_{22}\right)$ & $\overline{\mathbf{K}}_{2}^{0}$ \\
\cline { 2 - 4 } & 2 & $e_{2}^{2}=\frac{2}{3} \sqrt{\frac{3 \pi}{5}}\left(\mathrm{E}_{11}-\mathrm{E}_{22}\right)$ & $\overline{\mathbf{K}}_{2}^{2}$ \\
\hline
\end{tabular}

*The functions $\overline{\mathrm{K}}_{l}^{m}(\mathrm{~h})$ are the real spherical surface harmonics. (see Raymond et al.)

Table 2 Second-rank tensors. Polycrystals.

\begin{tabular}{|c|c|c|c|c|c|}
\hline \multicolumn{2}{|c|}{ Sample symmetry } & $l$ & $v$ & $\bar{e}_{l}^{v}$ & $\dot{K}_{l}^{\nu}(y)$ \\
\hline \multirow{5}{*}{\multicolumn{2}{|c|}{$C_{i}$}} & 0 & 1 & $e_{0}^{-1}=C_{0}^{11} e_{0}^{1}=e_{0}^{1}$ & $\overline{\mathbf{K}}_{0}^{0}$ \\
\hline & & 2 & 1 & $\begin{array}{l}\bar{e}_{2}^{-1}=\frac{1}{5}\left[C_{2}^{11} e_{2}^{1}+C_{2}^{21} e_{2}^{2}\right] \\
\bar{e}_{2}^{2}=\frac{1}{5}\left[\begin{array}{lll}C_{2}^{12} & e_{2}^{1}+C_{2}^{22} e_{2}^{2}\end{array}\right]\end{array}$ & $\begin{array}{l}\overline{\mathbf{K}}_{2}^{-2} \\
\overline{\mathbf{K}}_{2}^{-1}\end{array}$ \\
\hline & & & 3 & $\bar{e}_{2}^{-3}=\frac{1}{5}\left[C_{2}^{13} e_{2}^{1}+C_{2}^{23} e_{2}^{2}\right]$ & $\overline{\mathbf{K}}_{2}^{0}$ \\
\hline & & & 4 & & $\overline{\mathbf{K}}_{2}^{1}$ \\
\hline & & & 5 & $e_{2}^{-5}=\frac{1}{5}\left[C_{2}^{15} e_{2}^{1}+C_{2}^{25} e_{2}^{2}\right]$ & $\overline{\mathbf{K}}_{2}^{2}$ \\
\hline \multirow{6}{*}{$C_{2 \mathrm{~h}}$} & \multirow[t]{4}{*}{$2 \| \mathrm{z}$} & 0 & 1 & $\bar{e}_{0}^{-1}=\bar{e}_{0 t}^{1}$ & $\dot{\mathbf{K}}_{0 t}^{1}$ \\
\hline & & 2 & 1 & $\bar{e}_{2}^{1}=\bar{e}_{2 t}^{1}$ & \\
\hline & & & 2 & $\bar{e}_{2}^{2}=\bar{e}_{2 t}^{2}$ & $\begin{array}{l}\mathbf{R}_{2 t} \\
\dot{x}^{3}\end{array}$ \\
\hline & & & 3 & $\bar{e}_{2}^{3}=\bar{e}_{2 t}^{3}$ & $\dot{\mathrm{K}}_{2 t}^{5}$ \\
\hline & \multirow[t]{2}{*}{$2 \| Y$} & 0 & 1 & $\bar{e}_{0}^{1}=\bar{e}_{0 t}^{1}$ & $\dot{\mathbf{K}}_{0 t}^{1}$ \\
\hline & & 2 & $\begin{array}{l}1 \\
2 \\
3\end{array}$ & $\begin{array}{l}\bar{e}_{2}^{1}=\bar{e}_{2 t}^{1} \\
\bar{e}_{2}^{2}=\bar{e}_{3 t}^{2} \\
\bar{e}_{2}^{3}=\bar{e}_{2 t}^{3}\end{array}$ & $\begin{array}{l}\dot{\mathrm{K}}_{2 t}^{3} \\
\dot{\mathrm{K}}_{2 t}^{4} \\
\dot{\mathrm{K}}_{2 t}^{5}\end{array}$ \\
\hline
\end{tabular}


Table 2 Cont'd.

\begin{tabular}{c|c|c|c|c}
\hline Sample symmetry & $l$ & $v$ & $\bar{e}_{l}^{v}$ & $\dot{K}_{l}^{v}(y)$ \\
\hline \multirow{4}{*}{$D_{2 \mathrm{~h}}$} & 0 & 1 & $\bar{e}_{0}^{1}=\bar{e}_{0 t}^{1}$ & $\dot{\mathrm{K}}_{0 t}^{1}$ \\
\cline { 2 - 5 } & 2 & 1 & $\bar{e}_{2}^{1}=\bar{e}_{2 t}^{1}$ & $\dot{\mathrm{K}}_{2 t}^{3}$ \\
& & 2 & $\bar{e}_{2}^{2}=\bar{e}_{2 t}^{2}$ & $\dot{\mathrm{K}}_{2 t}^{5}$ \\
\hline
\end{tabular}

(In the present paper, subindex $t$ means triclinic.)

$$
d_{111}^{\prime}=\sum_{i, j, k}^{l} a_{1 i} a_{1 j} a_{1 k} d_{i j k}
$$

where the transformation components $a_{l i}$ are the direction cosines of the orientation $\mathrm{h}=\mathrm{OX}^{\prime}{ }_{1}$ relative to the coordinate axes $\mathrm{OX}_{i}$.

$e_{l}^{\mu}$ coefficients and $\dot{\mathrm{K}}_{l}^{\mu}(\mathrm{h})$ functions for expansion (1) corresponding to triclinic crystal symmetry are shown in Table 3. So-called reduced (matrix) notation of the independent components $d_{i j}$ is used. The non-centrosymmetric nature of the considered property implies that only odd- $l$ coefficients are non-null.

Table 4 gives $e_{l}^{\mu}$ for other crystal systems, with reference to those reported in Table 3. $\mathrm{K}_{l}^{\prime \mu}(\mathrm{h})$ functions follow the same relationship.

Table 3 Third-rank tensors. Single crystals. Triclinic symmetry.

\begin{tabular}{l|c|c|c}
\hline$l$ & $\mu$ & $e_{l}{ }^{\mu}$ & $\dot{\mathrm{K}}_{l}^{\mu}(h)$ \\
\hline 1 & 1 & $e_{1}^{1}=\frac{2}{5} \sqrt{\frac{\pi}{3}}\left[3 d_{22}+d_{21}+d_{23}+d_{16}+d_{34}\right]$ & $\overline{\mathbf{K}}_{1}^{-1}$ \\
\cline { 2 - 4 } & 2 & $e_{1}^{2}=\frac{2}{5} \sqrt{\frac{\pi}{3}}\left[3 d_{33}+d_{32}+d_{31}+d_{15}+d_{24}\right]$ & $\overline{\mathbf{K}}_{1}^{0}$ \\
\hline 3 & $e_{1}^{3}=\frac{2}{5} \sqrt{\frac{\pi}{3}}\left[3 d_{11}+d_{12}+d_{13}+d_{26}+d_{35}\right]$ & $\overline{\mathbf{K}}_{1}^{1}$ \\
\hline & 1 & $e_{3}^{1}=\sqrt{\frac{2 \pi}{35}}\left[d_{16}+d_{21}-d_{22}\right]$ & $\overline{\mathbf{K}}_{3}^{-3}$ \\
\cline { 2 - 4 } & 2 & $e_{3}^{2}=2 \sqrt{\frac{\pi}{105}}\left[d_{14}+d_{25}+d_{36}\right]$ & $\overline{\mathbf{K}}_{3}^{-2}$ \\
\cline { 2 - 4 } & 3 & $e_{3}^{3}=\frac{2}{5} \sqrt{\frac{\pi}{42}}\left[4\left(d_{23}+d_{34}\right)-\left(3 d_{22}+d_{21}+d_{16}\right)\right]$ & $\overline{\mathbf{K}}_{3}^{-1}$ \\
\cline { 2 - 4 } & 4 & $e_{3}^{4}=\frac{2}{5} \sqrt{\frac{\pi}{7}}\left[2 d_{33}-\left(d_{32}+d_{31}+d_{24}+d_{15}\right)\right]$ & $\overline{\mathbf{K}}_{3}^{0}$ \\
\hline
\end{tabular}


Table 3 Cont'd.

\begin{tabular}{l|c|c|c}
\hline$l$ & $\mu$ & $e_{l}{ }^{\mu}$ & $\dot{K}_{l}^{\mu}(h)$ \\
\hline 5 & $e_{3}^{5}=\frac{2}{5} \sqrt{\frac{\pi}{42}}\left[4\left(d_{13}+d_{35}\right)-\left(3 d_{11}+d_{12}+d_{26}\right)\right]$ & $\overline{\mathrm{K}}_{3}^{1}$ \\
\cline { 2 - 4 } & 6 & $e_{3}^{6}=2 \sqrt{\frac{\pi}{105}}\left[d_{15}+d_{31}-d_{24}-d_{32}\right]$ & $\overline{\mathrm{K}}_{3}^{2}$ \\
\cline { 2 - 4 } & 7 & $e_{3}^{7}=\sqrt{\frac{2 \pi}{35}}\left[d_{11}-d_{12}-d_{26}\right]$ & $\overline{\mathrm{K}}_{3}^{3}$ \\
\hline
\end{tabular}

Table 4 Third-rank tensors. Single crystals, Other symmetries.

\begin{tabular}{|c|c|c|c|c|}
\hline \multirow[t]{2}{*}{ Crystalline symmetry } & \multicolumn{2}{|c|}{ Class } & \multicolumn{2}{|c|}{$e_{l}^{\mu}$} \\
\hline & & & $l=1$ & $l=3$ \\
\hline \multirow{4}{*}{ Monoclinic } & \multirow[t]{2}{*}{$C_{2}$} & $2 \|_{x_{3}}$ & $e_{1}^{1}=e_{1 t}^{2}$ & $\begin{array}{l}e_{3}^{1}=e_{3 t}^{2} \\
e_{3}^{2}=e_{3 t}^{4} \\
e_{3}^{3}=e_{3 t}^{6}\end{array}$ \\
\hline & & $2 \|_{x_{2}}$ & $e_{1}^{1}=e_{1 t}^{1}$ & $\begin{array}{l}e_{3}^{1}=e_{3 t}^{1} \\
e_{3}^{2}=e_{3 t}^{2} \\
e_{3}^{3}=e_{3 t}^{3}\end{array}$ \\
\hline & \multirow[t]{2}{*}{$C_{s}$} & $m \perp x_{3}$ & $\begin{array}{l}e_{1}^{1}=e_{1 t}^{1} \\
e_{1}^{2}=e_{1 t}^{3}\end{array}$ & $\begin{array}{l}e_{3}^{1}=e_{3 t}^{1} \\
e_{3}^{2}=e_{3 t}^{3} \\
e_{3}^{3}=e_{3 t}^{5} \\
e_{3}^{4}=e_{3 t}^{7}\end{array}$ \\
\hline & & $m \perp x_{2}$ & $\begin{array}{l}e_{1}^{1}=e_{1 t}^{2} \\
e_{1}^{2}=e_{1 t}^{3}\end{array}$ & $\begin{aligned} e_{3}^{1} & =e_{3 t}^{4} \\
e_{3}^{2} & =e_{3 t}^{5} \\
e_{3}^{3} & =e_{3 t}^{6} \\
e_{3}^{4} & =e_{3 t}^{7}\end{aligned}$ \\
\hline \multirow[b]{2}{*}{ Orthorhombic } & \multicolumn{2}{|c|}{$\mathrm{D}_{2}$} & & $e_{3}^{1}=e_{3 t}^{2}$ \\
\hline & \multicolumn{2}{|c|}{$C_{2 v}$} & $e_{1}^{1}=e_{1 t}^{2}$ & $\begin{aligned} e_{3}^{1} & =e_{3 t}^{4} \\
e_{3}^{2} & =e_{3 t}^{6}\end{aligned}$ \\
\hline \multirow{5}{*}{ Tetragonal } & \multicolumn{2}{|c|}{$C_{4}$ and $C_{4 v}$} & $e_{1}^{1}=e_{1 t}^{2}$ & $\begin{array}{l}e_{3}^{1}=e_{3 t}^{4} \\
e_{3}^{2}=e_{3 t}^{6}\end{array}$ \\
\hline & \multicolumn{2}{|c|}{$S_{4}$} & & $\begin{array}{l}e_{3}^{1}=e_{3 t}^{2} \\
e_{3}^{2}=e_{3 t}^{6}\end{array}$ \\
\hline & \multicolumn{2}{|c|}{$D_{4}$} & \multicolumn{2}{|c|}{$d_{111}^{\prime} \equiv 0$} \\
\hline & \multirow[t]{2}{*}{$D_{2 \mathrm{~d}}$} & $m \perp x_{1}$ & & $e_{3}^{1}=e_{3 t}^{6}$ \\
\hline & & $2 \| x_{1}$ & & $e_{3}^{1}=e_{3 t}^{2}$ \\
\hline
\end{tabular}


Table 4 Cont'd.

\begin{tabular}{|c|c|c|c|c|}
\hline \multirow[t]{2}{*}{ Crystalline symmetry } & \multicolumn{2}{|c|}{ Class } & \multicolumn{2}{|c|}{$e_{l}^{\mu}$} \\
\hline & & & $l=1$ & $l=3$ \\
\hline \multirow{4}{*}{ Trigonal } & \multicolumn{2}{|c|}{$C_{3}$} & $e_{1}^{1}=e_{1 t}^{2}$ & $\begin{array}{l}e_{3}^{1}=e_{3 t}^{1} \\
e_{3}^{2}=e_{3 t}^{4} \\
e_{3}^{3}=e_{3 t}^{7}\end{array}$ \\
\hline & \multicolumn{2}{|c|}{$D_{3}$} & & $e_{3}^{1}=e_{3 t}^{7}$ \\
\hline & \multirow[t]{2}{*}{$C_{3 v}$} & $m \perp x_{2}$ & $e_{1}^{1}=e_{1 t}^{2}$ & $\begin{array}{l}e_{3}^{1}=e_{3 t}^{4} \\
e_{3}^{2}=e_{3 t}^{7}\end{array}$ \\
\hline & & $m \perp x_{1}$ & $e_{1}^{1}=e_{1 t}^{2}$ & $\begin{array}{l}e_{3}^{1}=e^{4}{ }_{3 t} \\
e^{2}{ }_{3}=e^{1}{ }_{3 t}\end{array}$ \\
\hline \multirow{5}{*}{ Hexagonal } & \multicolumn{2}{|c|}{$C_{6}$ and $C_{6 v}$} & $e_{1}^{1}=e_{1 t}^{2}$ & $e_{3}^{1}=e_{3 t}^{4}$ \\
\hline & \multicolumn{2}{|c|}{$D_{6}$} & \multicolumn{2}{|c|}{$d_{111}^{\prime} \equiv 0$} \\
\hline & \multicolumn{2}{|c|}{$C_{3 \mathrm{~h}}$} & & $\begin{array}{l}e_{3}^{1}=e_{3 t}^{1} \\
e_{3}^{2}=e_{3 t}^{7}\end{array}$ \\
\hline & \multirow[t]{2}{*}{$D_{3 \mathrm{~h}}$} & $m \perp x_{2}$ & & $e^{1}{ }_{3}=e^{7}{ }_{3 t}$ \\
\hline & & $m \perp x_{1}$ & & $e_{3}^{1}=e^{1}{ }_{3 t}$ \\
\hline \multirow[t]{2}{*}{ Cubic } & \multicolumn{2}{|c|}{$O$} & \multicolumn{2}{|c|}{$d_{i j} \equiv 0$} \\
\hline & \multicolumn{2}{|c|}{$T$ and $T_{\mathrm{d}}$} & & $e_{3}^{1}=e_{3 t}^{2}$ \\
\hline
\end{tabular}

For polycrystal average piezoelectricity one obtains, if sample symmetry is triclinic:

$$
\begin{aligned}
\bar{d}_{111}^{\prime}(\mathrm{y}) & =\bar{e}_{1}^{1} \dot{K}_{1}^{1}(\mathrm{y})+\bar{e}_{1}^{2} \dot{K}_{1}^{2}(\mathrm{y})+\bar{e}_{1}^{3} \dot{K}_{1}^{3}(\mathrm{y})+\bar{e}_{3}^{1} \dot{K}_{3}^{1}(\mathrm{y})+\bar{e}_{3}^{2} \dot{K}_{3}^{2}(\mathrm{y})+\bar{e}_{3}^{3} \dot{K}_{3}^{3}(\mathrm{y}) \\
& +\bar{e}_{3}^{4} \dot{K}_{3}^{4}(\mathrm{y})+\bar{e}_{3}^{5} \dot{K}_{3}^{5}(\mathrm{y})+\bar{e}_{3}^{6} \dot{K}_{3}^{6}(\mathrm{y})+\bar{e}_{3}^{7} \dot{K}_{3}^{7}(\mathrm{y})
\end{aligned}
$$

with $\bar{e}_{l}{ }_{l}$ coefficients calculated from (5). For example, if the crystal symmetry is $C_{s}$ or $D_{3}$, then these coefficients are given in Table 5 .

The functions $\dot{\mathrm{K}}_{l}^{v}$ (y) in (12) are the same as those reported in Table 3, with $v=\mu$. For other sample symmetries the average value of the property may be obtained from (12), using Table 6 . In these cases, the number of linearly independent functions depends on symmetry requirements (see Raymond et al.).

For fibre texture and any crystal symmetry one obtains

$$
\bar{d}_{111}^{\prime}(\phi)=\bar{e}_{1} \bar{P}_{1}(\phi)+\bar{e}_{3} \bar{P}_{3}(\phi)
$$


Table 5 Third-rank tensors. Coefficientes of the equation (12) for $C_{s}$ and $D_{3}$ symmetry.

\begin{tabular}{lll}
\hline & \multicolumn{1}{c}{$C_{3}$} & $D_{3}$ \\
\hline $\bar{e}_{1}^{v}(v=1,2,3)$ & $\frac{1}{3}\left[C_{1}^{1 v} e_{1}^{1}+C_{1}^{2 v} e_{1}^{2}\right]$ & 0 \\
\hline $\bar{e}_{3}^{v}(v=1,2, \ldots, 7)$ & $\frac{1}{7}\left[C_{3}^{1 v} e_{3}^{1}+C_{3}^{2 v} e_{3}^{2}+C_{3}^{3 v} e_{3}^{3}+C_{3}^{4 v} e_{3}^{4}\right]$ & $\frac{1}{7} C_{3}^{1 v} e_{3}^{1}$ \\
\hline
\end{tabular}

Table 6 Third-rank tensors. Polycrystals.

\begin{tabular}{|c|c|c|c|}
\hline \multirow{2}{*}{\multicolumn{2}{|c|}{ Sample symmetry }} & \multicolumn{2}{|c|}{$\dot{K}_{l}^{v}(y)$} \\
\hline & & $l=1$ & $l=3$ \\
\hline \multirow[t]{2}{*}{$C_{2}$} & $2 \| z$ & $\dot{\mathrm{K}}_{1}^{1}=\dot{\mathrm{K}}_{1 t}^{2}$ & $\begin{aligned} \dot{\mathrm{K}}_{3}^{1} & =\dot{\mathrm{K}}_{3 t}^{2} \\
\dot{\mathrm{K}}_{3}^{2} & =\dot{\mathrm{K}}_{3 t}^{4} \\
\dot{\mathrm{K}}_{3}^{3} & =\dot{\mathrm{K}}_{3 t}^{6}\end{aligned}$ \\
\hline & $2 \| Y$ & $\dot{\mathrm{K}}_{1}^{1}=\dot{\mathrm{K}}_{1 t}^{1}$ & $\begin{aligned} \dot{\mathbf{K}}_{3}^{1} & =\dot{\mathbf{K}}_{3 t}^{1} \\
\dot{\mathbf{K}}_{3}^{2} & =\dot{\mathbf{K}}_{3 t}^{2} \\
\dot{\mathbf{K}}_{3}^{3} & =\dot{\mathbf{K}}_{3 t}^{3}\end{aligned}$ \\
\hline \multirow[t]{2}{*}{$C_{s}$} & $m \perp Z$ & $\begin{aligned} \dot{\mathbf{K}}_{1}^{1} & =\dot{\mathbf{K}}_{1 t}^{1} \\
\dot{\mathrm{K}}_{1}^{2} & =\dot{\mathbf{K}}_{1 t}^{3}\end{aligned}$ & $\begin{aligned} \dot{\mathrm{K}}_{3}^{1} & =\dot{\mathrm{K}}_{3 t}^{1} \\
\dot{\mathrm{K}}_{3}^{2} & =\dot{\mathrm{K}}_{3 t}^{3} \\
\dot{\mathbf{K}}_{3}^{3} & =\dot{\mathbf{K}}_{3 t}^{5} \\
\dot{\mathbf{K}}_{3}^{4} & =\dot{\mathbf{K}}_{3 t}^{7}\end{aligned}$ \\
\hline & $m \perp \mathrm{Y}$ & $\begin{aligned} \dot{\mathbf{K}}_{1}^{1} & =\dot{\mathbf{K}}_{1 t}^{2} \\
\dot{\mathbf{K}}_{1}^{2} & =\dot{\mathbf{K}}_{1 t}^{3}\end{aligned}$ & $\begin{aligned} \dot{\mathrm{K}}_{3}^{1} & =\dot{\mathrm{K}}_{3 t}^{4} \\
\dot{\mathrm{K}}_{3}^{2} & =\dot{\mathbf{K}}_{3 t}^{5} \\
\dot{\mathrm{K}}_{3}^{3} & =\dot{\mathbf{K}}_{3 t}^{6} \\
\dot{\mathrm{K}}_{3}^{4} & =\dot{\mathbf{K}}_{3 t}^{7}\end{aligned}$ \\
\hline \multicolumn{2}{|c|}{$D_{2}$} & & $\dot{\mathbf{K}}_{3}^{1}=\dot{\mathbf{K}}_{3 t}^{2}$ \\
\hline \multicolumn{2}{|c|}{$C_{2 v}$} & $\dot{\mathbf{K}}_{1}^{1}=\dot{\mathbf{K}}_{1 t}^{2}$ & $\begin{aligned} \dot{\mathbf{K}}_{3}^{1} & =\dot{\mathbf{K}}_{3 t}^{4} \\
\dot{\mathbf{K}}_{3}^{2} & =\dot{\mathbf{K}}_{3 t}^{6}\end{aligned}$ \\
\hline
\end{tabular}

with $\bar{e}_{l}$ triclinic coefficients:

$$
\begin{aligned}
& \bar{e}_{1}=\frac{1}{4 \pi} \sqrt{\frac{2}{3}} \sum_{\mu=1}^{3} C_{1}{ }^{\mu} e_{1}^{\mu} \\
& \bar{e}_{3}=\frac{1}{4 \pi} \sqrt{\frac{2}{7}} \sum_{\mu=1}^{7} C_{3}^{\mu} e_{3}^{\mu}
\end{aligned}
$$


The number of terms in (14) and (15) for other symmetries will depend on the non-null $e_{l}^{\mu}$ reported in Table 4.

\section{TENSOR PROPERTIES OF FOURTH RANK}

Elasticity is the characteristic property associated to fourth rank tensors. It may also be described by longitudinal surfaces according to the transformation law:

$$
S_{1111}^{\prime}=\sum_{i, j, k, l} a_{l i} a_{1 j} a_{1 k} a_{1 l} S_{i j k l}
$$

If $S_{i j k l}$ is the compliance tensor, then the surface $S_{1111}^{\prime}$ gives the inverse of Young's modulus or eventually elasticity modulus. Expressions for $e_{l}^{\mu}-$ coefficients and $\dot{\mathbf{K}}_{l}^{\mu}(\mathrm{h})$ - functions are shown in Tables 7 and 8 for all crystal symmetries, excluding the cubic case. The reduced notation $S_{i j}$ is used. Due to centrosymmetric nature of the considered property, all odd-l coefficients in (1) and (4) are null.

Table 7 Fourth-rank tensors. Single crystals. Triclinic symmetry.

\begin{tabular}{l|l|l|l}
\hline$l$ & $\mu$ & \multicolumn{1}{|c|}{$e_{l}^{\mu}$} & $\dot{K}_{l}^{\mu}(h)$ \\
\hline 0 & 1 & $e_{0}^{1}=\frac{2}{5} \sqrt{\pi}\left[S_{11}+S_{22}+S_{33}+\frac{2}{3}\left(S_{12}+S_{13}+S_{23}\right)+\frac{1}{3}\left(S_{44}+S_{55}+S_{66}\right)\right]$ & $\overline{\mathbf{K}}_{0}^{0}$ \\
\hline 2 & 1 & $e_{2}^{1}=\frac{4}{35} \sqrt{15 \pi}\left[S_{16}+S_{26}+\frac{1}{3}\left(S_{36}+S_{45}\right)\right]$ & $\overline{\mathbf{K}}_{2}^{-2}$ \\
\cline { 2 - 5 } & 2 & $e_{2}^{2}=\frac{4}{105} \sqrt{15 \pi}\left[S_{14}+S_{56}+3\left(S_{24}+S_{34}\right)\right]$ & $\overline{\mathbf{K}}_{2}^{-1}$ \\
\hline \multirow{2}{*}{3} & $e_{2}^{3}=\frac{3}{105} \sqrt{5 \pi}\left[2\left(S_{66}+6 S_{33}+S_{13}+S_{23}\right)-\left(6 S_{11}+6 S_{22}+S_{44}+S_{55}+4 S_{12}\right)\right]$ & $\overline{\mathbf{K}}_{2}^{0}$ \\
\hline 4 & $e_{2}^{4}=\frac{4}{105} \sqrt{15 \pi}\left[S_{25}+S_{46}+3\left(S_{15}+S_{35}\right)\right]$ & $\overline{\mathbf{K}}_{2}^{1}$ \\
\hline \multirow{2}{*}{5} & $e_{2}^{5}=\frac{4}{35} \sqrt{15 \pi}\left[S_{11}-S_{22}+\frac{1}{3}\left(S_{13}-S_{23}\right)-\frac{1}{6}\left(S_{44}-S_{55}\right)\right]$ & $\overline{\mathbf{K}}_{2}^{2}$ \\
\hline 1 & $e_{4}^{1}=\frac{4}{105} \sqrt{35 \pi}\left[S_{16}-S_{26}\right]$ & $\overline{\mathbf{K}}_{4}^{-4}$ \\
\hline \multirow{2yyy}{*}{2} & $e_{4}^{3}=\frac{3}{105} \sqrt{70 \pi}\left[S_{14}+S_{56}-S_{24}\right]$ & $\overline{\mathbf{K}}_{4}^{-3}$ \\
\hline 3 & $e_{4}^{3}=\frac{4}{105} \sqrt{5 \pi}\left[2\left(S_{36}+S_{45}\right)-\left(S_{16}+S_{26}\right)\right]$ & $\overline{\mathbf{K}}_{4}^{-1}$ \\
\hline 4 & $e_{4}^{4}=\frac{6}{315} \sqrt{10 \pi}\left[4 S_{34}-\left(S_{14}+3 S_{24}+S_{56}\right)\right]$ & \\
\hline
\end{tabular}


Table 7 Cont'd

\begin{tabular}{l|l|c|c}
\hline$l$ & $\mu$ & \multicolumn{1}{|c|}{$e_{l}^{\mu}$} & $\dot{\mathrm{K}}_{l}^{\mu}(h)$ \\
\hline & 5 & $e_{4}^{5}=\frac{2}{105} \sqrt{\pi}\left[3\left(S_{11}+S_{22}\right)+8\left(S_{33}-S_{13}-S_{22}\right)-4\left(S_{44}+S_{55}+S_{66}+S_{12}\right)\right]$ & $\overline{\mathbf{K}}_{4}^{0}$ \\
\hline \multirow{2}{*}{6} & $e_{4}^{6}=\frac{6}{315} \sqrt{10 \pi}\left[4 S_{35}-\left(3 S_{15}+S_{25}+S_{46}\right)\right]$ & $\overline{\mathbf{K}}_{4}^{1}$ \\
\hline 7 & $e_{4}^{7}=\frac{4}{105} \sqrt{5 \pi}\left[2 S_{13}+S_{22}+S_{55}-S_{11}-S_{44}-2 S_{23}\right]$ & $\overline{\mathbf{K}}_{4}^{2}$ \\
\hline 8 & $e_{4}^{8}=\frac{2}{105} \sqrt{70 \pi}\left[S_{15}-\left(S_{25}+S_{46}\right)\right]$ & $\overline{\mathbf{K}}_{4}^{3}$ \\
\hline 9 & $e_{4}^{9}=\frac{4}{105} \sqrt{35 \pi}\left[S_{11}+S_{22}-S_{66}-2 S_{12}\right]$ & $\overline{\mathbf{K}}_{4}^{4}$ \\
\hline
\end{tabular}

Table 8 Fourth-rank tensors. Single crystals. Other symmetries.

\begin{tabular}{|c|c|c|c|c|c|}
\hline \multirow[t]{2}{*}{ Crystalline system } & \multirow{2}{*}{\multicolumn{2}{|c|}{ Class }} & \multicolumn{3}{|c|}{$e_{l}^{\mu}$} \\
\hline & & & $l=0$ & $l=2$ & $l=4$ \\
\hline \multirow[t]{2}{*}{ Monoclinic } & \multirow[t]{2}{*}{ all } & $2 \|_{x_{3}}$ & $e_{0}^{1}=e_{0 t}^{1}$ & $\begin{array}{l}e_{2}^{1}=e_{2 t}^{1} \\
e_{2}^{2}=e_{2 t}^{3} \\
e_{2}^{3}=e_{2 t}^{5}\end{array}$ & $\begin{aligned} e_{4}^{1} & =e_{4 t}^{1} \\
e_{4}^{2} & =e_{4 t}^{3} \\
e_{4}^{3} & =e_{4 t}^{5} \\
e_{4}^{4} & =e_{4 t}^{7} \\
e_{4}^{5} & =e_{4 t}^{9}\end{aligned}$ \\
\hline & & $2 \| x_{2}$ & $e_{0}^{1}=e_{0 t}^{1}$ & $\begin{array}{l}e_{2}^{1}=e_{2 t}^{3} \\
e_{2}^{2}=e_{2 t}^{4} \\
e_{2}^{3}=e_{2 t}^{5}\end{array}$ & $\begin{aligned} e_{4}^{1} & =e_{4 t}^{5} \\
e_{4}^{2} & =e_{4 t}^{6} \\
e_{4}^{3} & =e_{4 t}^{7} \\
e_{4}^{4} & =e_{4 t}^{8} \\
e_{4}^{5} & =e_{4 t}^{9}\end{aligned}$ \\
\hline Orthorhombic & & & $e_{0}^{1}=e_{0 t}^{1}$ & $\begin{array}{l}e_{2}^{1}=e_{2 t}^{3} \\
e_{2}^{2}=e_{2 t}^{5}\end{array}$ & $\begin{array}{l}e_{4}^{1}=e_{4 t}^{5} \\
e_{4}^{2}=e_{4 t}^{7} \\
e_{4}^{3}=e_{4 t}^{9}\end{array}$ \\
\hline \multirow[t]{2}{*}{ Tetragonal } & \multicolumn{2}{|c|}{$C_{4}, S_{4}, C_{4 h}$} & $e_{0}^{1}=e_{0 t}^{1}$ & $e_{2}^{1}=e_{2 t}^{3}$ & $\begin{array}{l}e_{4}^{1}=e_{4 t}^{1} \\
e_{4}^{2}=e_{4 t}^{5} \\
e_{4}^{3}=e_{4 t}^{9}\end{array}$ \\
\hline & \multicolumn{2}{|c|}{$D_{4}, C_{4 v}, D_{2 \mathrm{~d}}, D_{4 \mathrm{~h}}$} & $e_{0}^{1}=e_{0 t}^{1}$ & $e_{2}^{1}=e_{2 t}^{3}$ & $\begin{array}{l}e_{4}^{1}=e_{4 t}^{5} \\
e_{4}^{2}=e_{4 t}^{9}\end{array}$ \\
\hline
\end{tabular}


Table 8 Cont'd.

\begin{tabular}{|c|c|c|c|c|c|}
\hline \multirow{2}{*}{ Crystalline symmetry } & \multirow{2}{*}{\multicolumn{2}{|c|}{ Class }} & \multicolumn{3}{|c|}{$e_{l}^{\mu}$} \\
\hline & & & $l=0$ & $l=2$ & $l=4$ \\
\hline \multirow{3}{*}{ Trigonal } & \multicolumn{2}{|c|}{$C_{3}, C_{3 i}$} & $e_{0}^{1}=e_{0 t}^{1}$ & $e_{2}^{1}=e_{2 t}^{3}$ & $\begin{array}{l}e_{4}^{1}=e_{4 t}^{2} \\
e_{4}^{2}=e_{4 t}^{5}\end{array}$ \\
\hline & $D_{3}, C_{3 v}, D_{3 \mathrm{~d}}$ & $m \perp x_{1}$ & $e_{0}^{1}=e_{0 t}^{1}$ & $e_{2}^{1}=e_{2 t}^{3}$ & $\begin{array}{l}e_{4}^{1}=e_{4 t}^{5} \\
e_{4}^{2}=e_{4 t}^{2}\end{array}$ \\
\hline & $C_{3 v}, D_{3 \mathrm{~d}}$ & $m \perp x_{2}$ & $e_{0}^{1}=e_{0 t}^{1}$ & $e_{2}^{1}=e_{2 t}^{3}$ & $\begin{array}{l}e_{4}^{1}=e_{4 t}^{5} \\
e_{4}^{2}=e_{4 t}^{8}\end{array}$ \\
\hline Hexagonal & \multicolumn{2}{|l|}{ all } & $e_{0}^{1}=e_{0 t}^{1}$ & $e_{2}^{1}=e_{2 t}^{3}$ & $e_{4}^{1}=e_{4 t}^{5}$ \\
\hline
\end{tabular}

For cubic symmetry we obtain:

$$
\begin{aligned}
& e_{0}^{1}=\frac{2}{5} \sqrt{\pi}\left(3 S_{11}+2 S_{12}+S_{44}\right) \\
& e_{4}^{1}=\frac{4}{105} \sqrt{2 \pi}\left[2 S_{11}-\left(2 S_{12}+S_{44}\right)\right]
\end{aligned}
$$

with symmetric functions (see Raymond et al.):

$$
\begin{gathered}
\dot{\mathrm{K}}_{0}^{1}(\mathrm{~h})=\overline{\mathrm{K}}_{0}^{0}(\mathrm{~h}) \\
\dot{\mathrm{K}}_{4}^{1}(\mathrm{~h})=\sqrt{\frac{7}{24}}\left[\sqrt{2} \overline{\mathrm{K}}_{4}^{0}(\mathrm{~h})+\frac{\sqrt{70}}{7} \overline{\mathrm{K}}_{4}^{4}(\mathrm{~h})\right]
\end{gathered}
$$

For a polycrystal, it is possible to express the average elasticity in the form (12). For sample triclinic symmetry, then the expansion of function $\bar{S}_{11}^{\prime}(\mathrm{y})$ will have one term for $l=0$, five for $l=2$ and nine for $l=4$. Other sample symmetries are solved by the same method as that leading to Table 6, taking into account Tables 7 and 8 and using equations (4)-(5). For example, if crystal-sample symmetry is $D_{4 \mathrm{~h}}-D_{2 \mathrm{~h}}$ one obtains:

$$
\begin{aligned}
\bar{S}_{11}^{\prime}(\mathrm{y})= & \bar{e}_{0}^{1} \dot{\mathrm{K}}_{0 t}^{1}(\mathrm{y})+\bar{e}_{2}^{1} \dot{\mathrm{K}}_{2 t}^{3}(\mathrm{y})+\bar{e}_{2}^{2} \dot{\mathrm{K}}_{2 t}^{5}(\mathrm{y})+\bar{e}_{4}^{1} \dot{\mathrm{K}}_{4 t}^{5}(\mathrm{y})+\bar{e}_{4}^{2} \dot{\mathrm{K}}_{4 t}^{7}(\mathrm{y}) \\
& +\bar{e}_{4}^{3} \dot{\mathrm{K}}_{4 t}^{9}(\mathrm{y})
\end{aligned}
$$

with

$$
\bar{e}_{0}^{1}=C_{0}^{11} e_{0}^{1}=e_{0}^{1} \quad \bar{e}_{4}^{1}=\frac{1}{9}\left[C_{4}^{11} e_{4 t}^{5}+C_{4}^{21} e_{4 t}^{9}\right]
$$




$$
\begin{array}{ll}
\bar{e}_{2}^{1}=\frac{1}{5} C_{2}^{11} e_{2 t}^{3} & \bar{e}_{4}^{2}=\frac{1}{9}\left[C_{4}^{12} e_{4 t}^{5}+C_{4}^{22} e_{4 t}^{9}\right] \\
\bar{e}_{2}^{2}=\frac{1}{5} C_{2}^{12} e_{2 t}^{3} & \bar{e}_{4}^{3}=\frac{1}{9}\left[C_{4}^{13} e_{4 t}^{5}+C_{4}^{23} e_{4 t}^{9}\right]
\end{array}
$$

Function $\dot{\mathrm{K}}_{l t}^{v}(\mathrm{y})$ in (20) are the same as those reported in Table 7 for $v=\mu$.

Finally, for fibre textures

$$
\bar{S}_{11}^{\prime}(\mathrm{y})=\bar{e}_{0} \bar{P}_{0}(\phi)+\bar{e}_{2} \bar{P}_{2}(\phi)+\bar{e}_{4} \bar{P}_{4}(\phi)
$$

where $\bar{e}_{l}$ for triclinic crystal symmetry are given by:

$$
\begin{aligned}
& \bar{e}_{0}=\frac{\sqrt{2}}{4 \pi} C_{0}^{1} e_{0}^{1}=\frac{1}{\sqrt{2 \pi}} e_{0}^{1} \\
& \bar{e}_{2}=\frac{1}{4 \pi} \sqrt{\frac{2}{5}} \sum_{\mu=1}^{5} C_{2}^{\mu} e_{2}^{\mu} \\
& \bar{e}_{4}=\frac{1}{4 \pi} \sqrt{\frac{2}{9}} \sum_{\mu=1}^{9} C_{4}^{\mu} e_{4}^{\mu}
\end{aligned}
$$

The number of terms in (24)-(25) will depend, for the other crystal symmetries, on the non-null coefficients $e_{l}^{\mu}$ reported in Table 8.

\section{References}

Bunge, H. J., Roberts W. T. (1969). Orientation Distribution, Elastic and Plastic Anisotropy in Stabilized Steel Sheet. J. Appl. Cryst. Vol. 2, 116.

Bunge, H. J. (1982). Texture Analysis in Material Science. Butterworths, London.

Bunge, H. J. (1989). Texture and Magnetic Properties. Text and Microstruct, Vol. 11, pp. 75-91.

Humbert, M., Wagner, F., Philippe, M. J., Esling, C. (1991). Relation between texture and anisotropic properties: some applications to low symmetry materials. Text. and Microstruct., Vols 14-18, pp. 443-461.

Mainprice, D., Humbert, M. (1994). Methods of calculating petrophysical properties from lattice preferred orientation data. Surv. Geophys. Vol 15, pp. 575-592.

Matthies, S., Humbert, M., Schuman, C. (1994). On the use of the geometric mean approximation in residual stress analysis. Phys. Status Sol. (B). Vol 186, pp. K41-K44.

Nye, J. F. (1957). Physical Properties of Crystals: Their representation by tensors and matrices. Clarendon Press. Oxford.

Raymond, O., Fuentes, L. Gómez, J. I. Computer-oriented real spherical harmonics for texture and properties analyses. (to be published). 